\title{
INTERACTIVE INFINITE MARKOV PARTICLE SYSTEMS WITH JUMPS
}

\author{
By
}

\author{
Seiji Hiraba
}

\begin{abstract}
In [2] we investigated independent infinite Markov particle systems as measure-valued Markov processes with jumps, and we gave sample path properties and martingale characterizations. In particular, we investigated the exponent of Hölder-right continuity in case that the motion process is absorbing $\alpha$-stable motion on $(0, \infty)$ with $0<\alpha<2$, that is, time-changed absorbing Brownian motions on $(0, \infty)$ by the increasing $\alpha / 2$-stable Lévy processes.

In the present paper we shall extend the results to the case of simple interactive infinite Markov particle systems. We also consider the absorbing stable motion on a half space $H=\mathbf{R}^{d-1} \times(0, \infty)$ as a motion process.
\end{abstract}

\section{Settings and Previous Results}

In this section we give the general settings and the known results which are given in [2] in order to describe the main results in $\S 3$ and $\S 4$.

Let $S$ be a domain of $\mathbf{R}^{d}$. Let $\left(w(t), P_{x}\right)_{t \geq 0, x \in S}$ be a $S$-valued Markov process having life time $\zeta(w) \in(0, \infty]$ such that $w \in \mathbf{D}([0, \zeta(w)) \rightarrow S)$, i.e., $w:[0, \zeta(w)) \rightarrow S$ is right continuous and has left-hand limits. For convenience, we fix an extra point $\Delta \notin S$ and set $w(t)=\Delta$ if $t \geq \zeta(w)$. Moreover we shall extend functions $f$ on $S$ to on $\{\Delta\}$ by $f(\Delta)=0$, if necessary.

We use the following notations: Let $S \subset \mathbf{R}^{d}$ be a domain.

- If $x=\left(x_{1}, \ldots, x_{d}\right) \in \mathbf{R}^{d}$, then $\partial_{i_{1} \cdots i_{k}}^{k}=\partial^{k} /\left(\partial x_{i_{1}} \cdots \partial x_{i_{k}}\right), \partial_{i}^{k}=\partial^{k} /\left(\partial x_{i}^{k}\right)$ and $\partial_{i}=\partial_{i}^{1}$ for each $k=0,1, \ldots, i=1, \ldots, d$. Moreover $\partial_{t}=\partial / \partial t$ for time $t \geq 0$.

2000 Mathematics Subject Classification: Primary 60G57; Secondary 60G75.

Key words and phrases: particle systems, measure-valued processes, jump processes.

Received April 12, 2012.

Revised March 25, 2013. 
- $f \in C_{c} \equiv C_{c}(S) \stackrel{\text { def }}{\Longleftrightarrow} f$ is a continuous function on $S$ with compact support in $S$, and $C_{c}^{\infty} \equiv C_{c}^{\infty}(S):=C_{c}(S) \cap C^{\infty}(S)$.

- For each integer $k \geq 0, C_{b}^{k}:=\left.C_{b}^{k}\left(\mathbf{R}^{d}\right)\right|_{S}$, that is, $f \in C_{b}^{k} \stackrel{\text { def }}{\Longleftrightarrow} f$ is a restriction to $S$ of $k$-times continuously differentiable function on $\mathbf{R}^{d}$ with bounded derivatives of order between 0 and $k$. Moreover $f \in C_{0} \stackrel{\text { def }}{\Longleftrightarrow} f$ is continuous on $S$ and $f(x) \rightarrow 0$ whenever $x \rightarrow \partial S$ or $|x| \rightarrow \infty$. Furthermore $C_{b}:=C_{b}^{0}, C_{b}^{\infty}:=\bigcap_{k} C_{b}^{k}, C_{0}^{k}:=C_{0} \cap C_{b}^{k}$ and $C_{0}^{\infty}:=\bigcap_{k} C_{0}^{k}$.

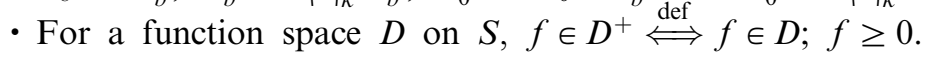

- $\langle\mu, f\rangle:=\int_{S} f(x) \mu(d x)$ for a function $f$ on $S$ and a measure $\mu$ on $S$.

The following two assumptions are the same as in [2].

Assumption 1. Let $\left(P_{t}\right)_{t \geq 0}$ be the transition semigroup of $\left(w(t), P_{x}\right)$, i.e., $P_{t} f(x)=E_{x}[f(w(t)): t<\zeta]$.

(i) $\left(P_{t}\right)$ is a strongly continuous nonnegative contraction semigroup on $\left(C_{0},\|\cdot\|_{\infty}\right)$ with generator $(A, \mathscr{D}(A))$, where $\|f\|_{\infty}=\sup _{x \in S}|f(x)|$.

(ii) $C_{c}^{\infty} \subset \mathscr{D}(A)$ and there is a strictly positive function $g_{0} \in C_{0}^{\infty}$ such that $g_{0} \in \mathscr{D}(A)$ and that $g_{0}^{-1} A f \in C_{b}$ with $g_{0}^{-1}=1 / g_{0}$ for every $f \in C_{c}^{\infty}$ and $f=g_{0}$.

(iii) $\sup _{t \leq T}\left\|g_{0}^{-1} P_{t} g_{0}\right\|_{\infty}<\infty$ for every $T>0$.

Under this assumption we introduce a function space $D_{g_{0}} \subset \mathscr{D}(A)$ as follows:

$$
f \in D_{g_{0}} \stackrel{\text { def }}{\Longleftrightarrow} f \in \mathscr{D}(A) \quad \text { such that }\left\|g_{0}^{-1} f\right\|_{\infty}<\infty \text { and }\left\|g_{0}^{-1} A f\right\|_{\infty}<\infty \text {. }
$$

Clearly $g_{0} \in D_{g_{0}}$ and $C_{c}^{\infty} \subset D_{g_{0}}$. Moreover since $C_{c}^{\infty}$ is dense in $C_{0}$ and $P_{t} C_{c}^{\infty} \subset D_{g_{0}}$ for every $t \geq 0, D_{g_{0}}$ is a core for $A$. However, $D_{g_{0}}$ may be too large, so we further need the following assumption:

Assumption 2. There exist a bounded function $g_{1} \in C^{\infty} ; g_{1} \geq g_{0}(>0)$ and a core $D \subset D_{g_{0}}$ (we denote $D=D_{g}$ with $g=\left(g_{0}, g_{1}\right)$ ) satisfying the following:

(i) If $f \in D_{g}$, then $\lim _{t \downarrow 0} \frac{1}{t}\left(P_{t} f^{2}(x)-f(x)^{2}\right)$ exists for each $x \in S$ (we also denote the limit as $\left.A f^{2}(x)\right), \quad \partial_{t} P_{t} f^{2}(x)=A P_{t} f^{2}(x)=P_{t} A f^{2}(x)$ $\left(\rightarrow A f^{2}(x)\right.$ as $t \downarrow 0$ for each $\left.x \in S\right), A f^{2} \in C_{b}$ and $\left\|g_{1}^{-1} A f^{2}\right\|_{\infty}<\infty$.

(ii) For each $T>0$, $\sup _{t \in[0, T]}\left\|g_{1}^{-1} P_{t} g_{1}\right\|_{\infty}<\infty$.

(iii) For each $0<s<T$, $\sup _{t \in[s, T]}\left\|g_{0}^{-1} P_{t} g_{1}\right\|_{\infty}<\infty$.

(iv) There exist constants $0 \leq \gamma<1, \delta>0$ such that $\sup _{0 \leq t \leq \delta} t^{\gamma}\left\|g_{0}^{-1} P_{t} g_{1}\right\|_{\infty}$ $<\infty$.

(v) $g_{0} \in D_{g}$. 
All through the present paper we suppose that Assumption 1 and 2 are fulfilled. We shall sometime use the notation $\|\cdot\|_{g_{0}}=\left\|\cdot / g_{0}\right\|_{\infty}$.

Let $\mathscr{M}_{g_{0}} \equiv \mathscr{M}_{g_{0}}(S)$ be a space of counting measures on $S$ defined as $\mu \in \mathscr{M}_{g_{0}} \stackrel{\text { def }}{\Longleftrightarrow} \mu=\sum_{n} \delta_{x_{n}}$ such that $\left\langle\mu, g_{0}\right\rangle<\infty$ and

$$
\begin{aligned}
& \mu_{n} \rightarrow \mu \text { in } \mathscr{M}_{g_{0}} \stackrel{\text { def }}{\Longleftrightarrow} \sup \left\langle\mu_{n}, g_{0}\right\rangle<\infty, \\
& \left\langle\mu_{n}, f\right\rangle \rightarrow\langle\mu, f\rangle \text { for all } f \in C_{c} \text { and } f=g_{0},
\end{aligned}
$$

where $C_{c} \equiv C_{c}(S)$ denotes the space of all continuous functions with compact supports on $S$. Then $\mathscr{M}_{g_{0}}$ is metrizable and separable.

We mainly consider the case that the generator has the form

$$
A=A^{c}+A^{d}
$$

with

$$
\begin{aligned}
A^{c} f(x)= & \frac{1}{2} \sum_{i, j=1}^{d} a^{i j}(x) \partial_{i j}^{2} f(x)+\sum_{i=1}^{d} b^{i}(x) \partial_{i} f(x), \\
A^{d} f(x)= & \int_{S \backslash\{x\}}[f(y)-f(x)-\nabla f(x) \cdot(y-x) I(|y-x|<1)] v(x, d y) \\
& -k(x) f(x)+\sum_{i=1}^{d} c^{i}(x) \partial_{i} f(x)
\end{aligned}
$$

for $f \in D_{g}$, where $a^{i j}, b^{i} \in C_{b}(S),\left(a^{i j}\right)$ is positive definite, $k(x) \geq 0$ denotes the killing rate by jumps, $\left(c^{i}(x)\right)$ depends on jumps, and $v(x, d y)$ is the Lévy kernel on $S \times(S \backslash\{x\})$ satisfying that

$$
\sup _{x \in S} \int_{S \backslash\{x\}}\left(1 \wedge|y-x|^{2}\right) v(x, d y)<\infty .
$$

\section{Independent IMPS; Results in [2]}

Let $\left(X_{t}, \mathbf{P}_{\mu}\right)$ be an (indistinguishable) independent Markov particle system (IMP) associated with the motion process $\left(w(t), P_{x}\right)$, i.e., for many independent motions $\left(w_{n}(t), P_{x_{n}}\right) \stackrel{(\mathrm{d})}{=}\left(w(t), P_{x_{n}}\right)$,

$$
X_{t}=\left.\sum_{n} \delta_{w_{n}(t)}\right|_{S} \quad \text { if } \mu=\sum_{n} \delta_{x_{n}} \text { on } S, \quad \text { and } \quad \mathbf{P}_{\mu}=\prod_{n} P_{x_{n}} .
$$


The generator $\mathscr{L}_{0}$ of this particle system is given by the following: for $f \in C_{c}^{\infty}$,

$$
\begin{aligned}
\mathscr{L}_{0} e^{-\langle\cdot, f\rangle}(\mu) & =-\left\langle\mu, e^{f} A\left(1-e^{-f}\right)\right\rangle e^{-\langle\mu, f\rangle} \\
& =-\langle\mu, A f-\Gamma f\rangle e^{-\langle\mu, f\rangle},
\end{aligned}
$$

where $\Gamma f:=A f-e^{f} A\left(1-e^{-f}\right)$. In fact, let $\left\{\mathscr{F}_{t}\right\}$ be the filtration generated by $\left\{X_{t}\right\}$ and set

$$
V_{t} f(x)=-\log P_{x}[\exp -f(w(t))]=-\log \left\{1-P_{t}\left(1-e^{-f}\right)(x)\right\} .
$$

We have that if $0 \leq s<t$, then

$$
\mathbf{E}_{\mu}\left[e^{-\left\langle X_{t}, f\right\rangle} \mid \mathscr{F}_{s}\right]=\exp \left[-\left\langle X_{s}, V_{t-s} f\right\rangle\right] .
$$

It is easy to see that $\left(V_{t}\right)_{t \geq 0}$ is a nonnegative contraction semigroup on $C_{0}$ and that by (ii) of Assumption 1 if $f \in C_{c}^{\infty}$, then $1-e^{-f} \in C_{c}^{\infty} \subset D_{g}$, hence we have

$$
\begin{gathered}
\partial_{t} V_{t} f=\frac{P_{t} A\left(1-e^{-f}\right)}{1-P_{t}\left(1-e^{-f}\right)}=\frac{A P_{t}\left(1-e^{-f}\right)}{1-P_{t}\left(1-e^{-f}\right)}=e^{V_{t} f} A\left(1-e^{-V_{t} f}\right) \\
\rightarrow e^{f} A\left(1-e^{-f}\right)=A f-\Gamma f \quad(t \downarrow 0) .
\end{gathered}
$$

Note that since $V_{t} f \leq P_{t} f$ (by Jensen's inequality), $\Gamma$ is nonnegative;

$$
\Gamma f=A f-\left.\partial_{t} V_{t} f\right|_{t=0+}=\lim _{t \downarrow 0} \frac{1}{t}\left[\left(P_{t} f-f\right)-\left(V_{t} f-f\right)\right] \geq 0
$$

and that for each $f \in C_{c}^{\infty}, v_{t}=V_{t} f$ is the unique solution to the following equation:

$$
\partial_{t} v_{t}=e^{v_{t}} A\left(1-e^{-v_{t}}\right), \quad v_{0}=f
$$

(because $u_{t}:=1-e^{-v_{t}}$ satisfies $\partial_{t} u_{t}=A u_{t}, u_{0}=1-e^{-f}$ and the unique solution is given as $\left.u_{t}=P_{t}\left(1-e^{-f}\right)\right)$. Moreover if $A v_{t}(x)$ is well-defined for $t>0, x \in S$, then

$$
\partial_{t} v_{t}=A v_{t}-\Gamma v_{t}, \quad v_{0}=f
$$

By using the Markov property and by induction we have

Proposition 1 (Prop. 1 in [1]). For every $0 \leq t_{1} \leq \cdots \leq t_{n}$ and $f_{i} \in D_{g}^{+}$, $i=1,2, \ldots, n$, 


$$
\begin{aligned}
& \mathbf{E}_{\mu}\left[\left\langle X_{t_{1}}, f_{1}\right\rangle \cdots\left\langle X_{t_{n}}, f_{n}\right\rangle\right] \\
& \leq \prod_{i=1}^{n}\left\langle\mu, P_{t_{i}} f_{i}\right\rangle+C_{1}^{(n)} \sum_{i=1}^{n} \prod_{j \neq i}\left\langle\mu, P_{t_{j}} f_{j}\right\rangle \\
& \quad+C_{2}^{(n)} \sum_{i_{1} \neq i_{2}} \prod_{j \neq i_{1}, i_{2}}\left\langle\mu, P_{t_{j}} f_{j}\right\rangle+\cdots+C_{n-1}^{(n)} \sum_{j=1}^{n}\left\langle\mu, P_{t_{j}} f_{j}\right\rangle+C_{n}^{(n)},
\end{aligned}
$$

where $C_{k}^{(n)}, k=1, \ldots, n$ are positive constants, depending on $\left(n,\left\{\left\|f_{i}\right\|_{\infty}\right\}_{i \leq n}\right)$.

We introduce a non-negative operator $Q$ as $Q f=A f^{2}-2 f A f$ for $f \in D_{g}$, which is well-defined by (i) of Assumption 2 and plays an important role to investigate the exponents of Hölder (right) continuity. The non-negativity follows from $\left(P_{t} f^{2}-f^{2}\right)-2 f\left(P_{t} f-f\right) \geq\left(P_{t} f\right)^{2}-2 f P_{t} f+f^{2}=\left(P_{t} f-f\right)^{2} \geq 0$.

TheOREM 1 (Th. 2.3 and Cor. 2.1 in [2]). Let $\left(w(t), P_{x}\right)$ be a discontinuous Markov process in $\mathbf{D}([0, \zeta(w)) \rightarrow S)$ with transition semigroup $\left(P_{t}\right)$ satisfying Assumption 1 and 2. Let $\mu \in \mathscr{M}_{g_{0}}$. The following holds with $\mathbf{P}_{\mu}$-probability one.

(i) $\left\{\left\langle X_{t}, g_{0}\right\rangle\right\}$ is $((1-\gamma) / 2-\varepsilon)$-Hölder right continuous at $t=0$ for suffciently small $\varepsilon>0$, where the constant $0 \leq \gamma<1$ is in (iv) of Assumption 2.

(ii) If $\left\langle\mu, g_{1}\right\rangle<\infty$, in particular, if $g_{1}(x)=g_{0}(x)$ then $\left\{\left\langle X_{t}, g_{0}\right\rangle\right\}$ is $(1 / 2-\varepsilon)$-Hölder right continuous at $t=0$ for sufficiently small $\varepsilon>0$.

(iii) For each fixed $t_{0}>0,\left\{\left\langle X_{t}, g_{0}\right\rangle\right\}$ is $(1 / 2-\varepsilon)$-Hölder right continuous at $t=t_{0}$ for sufficiently small $\varepsilon>0$.

\section{Sampling Replacement Markov Particle Systems}

Let $\mu=\sum_{n} \delta_{x_{n}} \in \mathscr{M}_{g_{0}}$. Let $\left(Y_{t}, \mathbf{P}_{\mu}^{Y}\right)$ be a sampling replacement Markov particle system associated with the motion process $\left(w(t), P_{x}\right)$, sampling replacement rate $\lambda>0$ and sampling replacement probability $q(d(m, n))=$ $\sum_{k, l} p_{k, l} \delta_{(k, l)}(d(m, n))$ on $\mathbf{N}^{2}$, where $p_{k, l} \geq 0, \quad p_{k, k}=0$ and $\sum p_{k, l}=1$. Each particle first moves independently each other. After a $\lambda$-exponential random time, two particles are selected randomly, for example, $m$-th and $n$-th particles are selected with probability $p_{m, n}$, and at that time the $m$-th particle jumps to the place of the $n$-th particle. Then the $m$-th particle moves independently. And these operations are continued. We denote each particle by $w_{n}^{*}(t)$ such that $w_{n}^{*}(0)=x_{n}$, and hence $Y_{t}=\sum_{n} \delta_{w_{n}^{*}(t)}$. Note that if $\left(P_{t}\right)$ is non-conservative, then it is possible that the dead particles come life again. 
Recall $\left(X_{t}, \mathbf{P}_{\mu}\right)$ is the independent Markov particle system with the motion process $\left(w(t), P_{x}\right)$. For $f \in D_{g}$, set $L_{t}^{Y}(\mu)=\mathbf{E}_{\mu}^{Y}\left[\exp -\left\langle Y_{t}, f\right\rangle\right]$ and $L_{t}(\mu)=$ $\mathbf{E}_{\mu}\left[\exp -\left\langle X_{t}, f\right\rangle\right]$. Then

$$
L_{t}(\mu)=e^{-\left\langle\mu, V_{t} f\right\rangle} \quad \text { with } V_{t} f(x)=-\log E_{x}\left[e^{-f(w(t))}\right]=-\log \left(1-P_{t}\left(1-e^{-f}\right)\right) .
$$

It is easy to see that $L_{t}^{Y}(\mu)$ satisfies the following equation:

$$
L_{t}^{Y}(\mu)=e^{-\lambda t} L_{t}(\mu)+\lambda \int_{0}^{t} d s e^{-\lambda s} \int_{\mathbf{N}^{2}} q(d(m, n)) \mathbf{P}_{s}\left(\Theta_{m, n} L_{t-s}\right)(\mu),
$$

where $\left(\mathbf{P}_{t}\right)$ is the transition semigroup of $\left(X_{t}, \mathbf{P}_{\mu}\right)$ and $\Theta_{m, n}$ is an operator such that it makes the $m$-th particle jump to the place of $n$-th particle of $\mu=$ $\sum \delta_{k} \in \mathscr{M}_{g_{0}}$ on a class of all functions $F(\mu)$ and it is defined by $\Theta_{m, n} F(\mu)=$ $F\left(\mu^{m, n}\right)$ with $\mu^{m, n}=\mu-\delta_{x_{m}}+\delta_{x_{n}}$. Note that $\mathbf{P}_{s} \Theta_{m, n}=\Theta_{m, n} \mathbf{P}_{s}$ holds. The solution is given as

$$
L_{t}^{Y}(\mu)=\mathbf{T}_{t} e^{-\left\langle\cdot, V_{t} f\right\rangle}(\mu) \quad \text { with } \mathbf{T}_{t}=\sum_{k} e^{-\lambda t} \frac{(\lambda t)^{k}}{k !}\left(\int_{\mathbf{N}^{2}} q(d(m, n)) \Theta_{m, n}\right)^{k}
$$

where $\mathbf{T}_{t}$ is an operator on a class of functions $F(\mu)$ with polynomial growth of $\left\langle\mu, f_{1}\right\rangle,\left\langle\mu, f_{2}\right\rangle, \ldots,\left\langle\mu, f_{n}\right\rangle\left(f_{i} \in D_{g}\right)$ and

$$
\left(\int_{\mathbf{N}^{2}} q(d(m, n)) \Theta_{m, n}\right)^{k} F(\mu)=\left(\int_{\mathbf{N}^{2}} q(d(m, n)) \Theta_{m, n}\right)^{k-1} \sum_{m, n \in \mathbf{N}} p_{m, n} F\left(\mu^{m, n}\right) .
$$

The generator $\mathscr{L}^{Y}$ of this particle system is given by the following: for $f \in C_{c}^{\infty}$,

$$
\begin{aligned}
& \mathscr{L}^{Y} e^{-\langle\cdot, f\rangle}(\mu)=\mathscr{L}_{0} e^{-\langle\cdot, f\rangle}(\mu)+\lambda \int\left(e^{-\left\langle\mu^{m, n}, f\right\rangle}-e^{-\langle\mu, f\rangle}\right) q(d(m, n)) \\
&=-\left\{\langle\mu, A f-\Gamma f\rangle+\lambda \int\left\langle\delta_{x_{n}}-\delta_{x_{m}}, f\right\rangle q(d(m, n))\right. \\
&\left.-\lambda \int\left[e^{-\left\langle\delta_{x_{n}}-\delta_{x_{m}}, f\right\rangle}-1+\left\langle\delta_{x_{n}}-\delta_{x_{m}}, f\right\rangle\right] q(d(m, n))\right\} e^{-\langle\mu, f\rangle}
\end{aligned}
$$

(more general formula of $\mathscr{L}^{Y} F(\mu)$ is given in $\S 5$ ). We have the following result. Recall that we denote the particles of $Y_{t}$ by $w_{n}^{*}(t)$, i.e., $Y_{t}=\sum \delta_{w_{n}^{*}(t)}$. Note that $w_{n}^{*}(t)$ moves like as $w_{n}(t)$ during the jump times. 
THEOREM 2 (Semi-martingale Representation of $Y_{t}$ ). Under Assumption 1 and 2 for $\left(P_{t}\right)$, if $\mu \in \mathscr{M}_{g_{0}}$, then $\left(Y_{t}, \mathbf{P}_{\mu}^{Y}\right)$ is an $\mathscr{M}_{g_{0}}$ valued Markov process with sample paths in $\mathbf{D}\left([0, \infty) \rightarrow \mathscr{M}_{g_{0}}\right)$ satisfying the following:

(i) $\left\{\left\langle Y_{t}, g_{0}\right\rangle\right\}$ has the same exponent of Hölder right continuity as in Theorem 1.

(ii) If the motion process $\left(w(t), P_{x}\right)$ has generator $A$ of the form as in (1.1), then for $f \in D_{g}$,

$$
\begin{aligned}
\left\langle Y_{t}, f\right\rangle= & \left\langle Y_{0}, f\right\rangle+\int_{0}^{t}\left\{\left\langle Y_{s}, A f\right\rangle+\lambda \int\left\langle\delta_{w_{n}^{*}(s)}-\delta_{w_{m}^{*}(s)}, f\right\rangle q(d(m, n))\right\} d s \\
& +M_{t}^{c}(f)+M_{t}^{d}(f)
\end{aligned}
$$

where

$$
M_{t}^{c}(f) \text { is a continuous } L^{2} \text {-martingale }
$$

with quadratic variation $\left\langle\left\langle M^{c}(f)\right\rangle\right\rangle_{t}=\int_{0}^{t}\left\langle Y_{s}, Q^{c} f\right\rangle d s=2 \int_{0}^{t}\left\langle Y_{s}, \Gamma^{c} f\right\rangle d s$ and

$$
M_{t}^{d}(f)=\int_{0}^{t} \int_{\mathscr{M}_{g_{0}}^{ \pm}}\langle\mu, f\rangle \tilde{N}(d s, d \mu) \quad \text { is a purely discontinuous martingale }
$$

where $\tilde{N}=N-\hat{N}$ is the martingale measure with

$$
\begin{aligned}
& N(d s, d \mu)=\sum_{u ; \Delta Y_{u} \neq 0} \delta_{\left(u, \Delta Y_{u}\right)}(d s, d \mu): \text { the jump measure of }\left\{Y_{t}\right\} \\
& \hat{N}(d s, d \mu)=d s\left\{\int Y_{s}(d x)\left(\int v(x, d y) \delta_{\left(\delta_{y}-\delta_{x}\right)}+k(x) \delta_{-\delta_{x}}\right)\right. \\
& \left.+\lambda \int q(d(m, n)) \delta_{\left(\delta_{w_{n}^{*}(s)}-\delta_{w_{m}^{*}(s)}\right)}\right\}(d \mu): \text { the compensator of } N .
\end{aligned}
$$

Proof. The proof is the same as the independent case (Proof of Theorem 2.4 in [2]). However, we need some computations. First the Markov property can be shown by mathematical induction. For $t_{1}<t_{2}, f_{1}, f_{2} \in C_{c}^{\infty}$, let $L_{t_{1}, t_{2}}^{f_{1}, f_{2}}(\mu)=$ $\mathbf{E}_{\mu}^{Y}\left[\exp \left(-\left\langle Y_{t_{1}}, f_{1}\right\rangle-\left\langle Y_{t_{2}}, f_{2}\right\rangle\right)\right]$. Recall that $L_{t}^{Y}(\mu)=L_{t}^{f}(\mu)=\mathbf{E}_{\mu}^{Y}\left[\exp -\left\langle Y_{t}, f\right\rangle\right]$ satisfies (2.1) and the solution is given as $L_{t}^{f}(\mu)=\mathbf{T}_{t} \mathbf{P}_{t}[\exp -\langle\cdot, f\rangle](\mu)$. Hence it is easy to see that $L_{t_{1}, t_{2}}^{f_{1}, f_{2}}(\mu)$ satisfies the following equation:

$$
\begin{aligned}
L_{t_{1}, t_{2}}^{f_{1}, f_{2}}(\mu)= & e^{-\lambda t_{1}} \mathbf{P}_{t_{1}}\left(e^{-\left\langle\cdot, f_{1}\right\rangle} L_{t_{2}-t_{1}}^{f_{2}}\right)(\mu) \\
& +\lambda \int_{0}^{t_{1}} d s e^{-\lambda s} \int q(d(m, n)) \mathbf{P}_{s}\left(\Theta_{m, n} L_{t_{1}-s, t_{2}-s}^{f_{1}, f_{2}}\right)(\mu) .
\end{aligned}
$$


The solution is given as

$$
\begin{aligned}
L_{t_{1}, t_{2}}^{f_{1}, f_{2}}(\mu) & =\mathbf{T}_{t_{1}} \mathbf{P}_{t_{1}}\left(e^{-\left\langle\cdot, f_{1}\right\rangle} L_{t_{2}-t_{1}}^{f_{2}}\right)(\mu) \\
& =\mathbf{E}_{\mu}^{Y}\left[e^{-\left\langle Y_{t_{1}}, f_{1}\right\rangle} \mathbf{E}_{Y_{t_{1}}}^{Y}\left[e^{-\left\langle Y_{t_{2}-t_{1}}, f_{2}\right\rangle}\right]\right] .
\end{aligned}
$$

Therefore by induction, for every $n \in \mathbf{N}$, if $t_{1}<t_{2}<\cdots<t_{n}, f_{1}, \ldots, f_{n} \in C_{c}^{\infty}$, then it holds that

$$
\begin{aligned}
& \mathbf{E}_{\mu}^{Y}\left[\exp \left(-\left\langle Y_{t_{1}}, f_{1}\right\rangle-\cdots-\left\langle Y_{t_{n}}, f_{n}\right\rangle\right)\right] \\
& \quad=\mathbf{E}_{\mu}^{Y}\left[e^{-\left\langle Y_{t_{1}}, f_{1}\right\rangle} \mathbf{E}_{Y_{t_{1}}}^{Y}\left[e^{-\left\langle Y_{t_{2}-t_{1}}, f_{2}\right\rangle} \cdots \mathbf{E}_{Y_{t_{n-1}}}^{Y}\left[e^{-\left\langle Y_{t_{n}-t_{n-1}}, f_{n}\right\rangle}\right] \cdots\right]\right] .
\end{aligned}
$$

Next we shall show that $\left(Y_{t}, \mathbf{P}_{\mu}^{Y}\right)$ satisfies a moment inequality of the same type as in Proposition 1.

Proposition 2. Let $T>0$ and $n \in \mathbf{N}$. For every $0 \leq t_{1} \leq \cdots \leq t_{n} \leq T$ and $f_{i} \in D_{g}^{+}, i=1,2, \ldots, n$,

$$
\begin{aligned}
\mathbf{E}_{\mu}^{Y}\left[\left\langle Y_{t_{1}}, f_{1}\right\rangle \cdots\left\langle Y_{t_{n}}, f_{n}\right\rangle\right] \\
\leq \prod_{i=1}^{n}\left\langle\mu, P_{t_{i}} f_{i}\right\rangle+C_{1, T}^{(n)} \sum_{i=1}^{n} \prod_{j \neq i}\left\langle\mu, P_{t_{j}} f_{j}\right\rangle \\
\quad+C_{2, T}^{(n)} \sum_{i_{1} \neq i_{2}} \prod_{j \neq i_{1}, i_{2}}\left\langle\mu, P_{t_{j}} f_{j}\right\rangle+\cdots+C_{n-1, T}^{(n)} \sum_{j=1}^{n}\left\langle\mu, P_{t_{j}} f_{j}\right\rangle+C_{n, T}^{(n)},
\end{aligned}
$$

where $C_{k, T}^{(n)}, k=1, \ldots, n$ are positive constants depending on $\left(n, T,\left\{\left\|f_{i}\right\|_{\infty}\right\}_{i \leq n}\right)$.

Proof. For simplicity, we use notations $f_{m}=f\left(x_{m}\right)$ and $\|\cdot\|=\|\cdot\|_{\infty}$. Since $\Theta_{m, \ell}\langle\mu, f\rangle=\langle\mu, f\rangle+f_{\ell}-f_{m} \leq\langle\mu, f\rangle+\|f\|$, we have for every $k \in \mathbf{N}$,

$$
\left(\int_{\mathbf{N}^{2}} q(d(m, \ell)) \Theta_{m, \ell}\right)^{k}\left(\left\langle\mu, f_{1}\right\rangle \cdots\left\langle\mu, f_{n}\right\rangle\right) \leq\left(\left\langle\mu, f_{1}\right\rangle+k\left\|f_{1}\right\|\right) \cdots\left(\left\langle\mu, f_{n}\right\rangle+k\left\|f_{n}\right\|\right) .
$$

Moreover if we denote by $M(j ; \lambda t)$ the $j$-th moment of $\lambda t$-Poisson distribution, then

$$
\begin{aligned}
& \mathbf{T}_{t}\left(\left\langle\cdot, f_{1}\right\rangle \cdots\left\langle\cdot, f_{n}\right\rangle\right)(\mu) \\
& \quad=\sum_{k \geq 0} e^{-\lambda t} \frac{(\lambda t)^{k}}{k !}\left(\int_{\mathbf{N}^{2}} q(d(m, \ell)) \Theta_{m, \ell}\right)^{k}\left(\left\langle\cdot, f_{1}\right\rangle \cdots\left\langle\cdot, f_{n}\right\rangle\right)(\mu) \\
& \quad \leq \sum_{j=0}^{n} M(n-j ; \lambda t) \sum_{\left\{i_{1}, \ldots, i_{j}\right\} \subset\{1, \ldots, n\}}\left\langle\mu, f_{i_{1}}\right\rangle \cdots\left\langle\mu, f_{i_{j}}\right\rangle\left\|f_{i_{j+1}}\right\| \cdots\left\|f_{i_{n}}\right\| .
\end{aligned}
$$


Therefore by applying Proposition 1 and the above inequality to

$$
\mathbf{E}_{\mu}^{Y}\left[\left\langle Y_{t_{1}}, f_{1}\right\rangle \cdots\left\langle Y_{t_{n}}, f_{n}\right\rangle\right]=\mathbf{T}_{t}\left(\mathbf{E} \cdot\left[\left\langle X_{t_{1}}, f_{1}\right\rangle \cdots\left\langle X_{t_{n}}, f_{n}\right\rangle\right]\right)(\mu)
$$

we can get the desired inequality.

Thus the proof can be proceeded by the same way as in the independent case (see $\S 4$ in [2]). In that way we can get the following result. For $\mu=\sum_{n} \delta_{x_{n}} \in \mathscr{M}_{g_{0}}$, $f \in C_{c}^{\infty}$, let

$$
\Psi(\mu ; f)=\langle\mu, A f-\Gamma f\rangle-\lambda \int\left[e^{-\left\langle\delta_{x_{m}}-\delta_{x_{n}}, f\right\rangle}-1\right] q(d(m, n)) .
$$

THEOREM 3. For $f \in C_{c}^{\infty}$,

$$
e^{-\left\langle Y_{t}, f\right\rangle}-e^{-\left\langle Y_{0}, f\right\rangle}-\int_{0}^{t} \mathscr{L}^{Y} e^{-\langle\cdot f\rangle}\left(Y_{S}\right) d s
$$

is a $\mathbf{P}_{\mu}^{Y}$-martingale. Moreover

$$
H_{t}(f)=\exp \left[-\left\langle Y_{t}, f\right\rangle+\int_{0}^{t} \Psi\left(Y_{s} ; f\right) d s\right]
$$

is also a $\mathbf{P}_{\mu}^{Y}$-martingale.

Proof. By the same way as in the proof of Theorem 4.1 in [2] we have if $s<t$, then

$$
\begin{aligned}
\partial_{t} \mathbf{E}_{\mu}^{Y}\left[e^{\left.-\left\langle Y_{t}, f\right\rangle \mid \mathscr{F}_{s}\right]}\right. & =\partial_{t} \mathbf{T}_{t-s}\left(e^{-\left\langle\cdot, V_{t-s} f\right\rangle}\right)\left(Y_{s}\right) \\
& =\partial_{u=0+} \mathbf{T}_{t-s+u}\left(e^{-\left\langle\cdot, V_{t-s+u} f\right\rangle}\right)\left(Y_{s}\right) \\
& =\partial_{u=0+} \mathbf{E}_{\mu}^{Y}\left[\mathbf{T}_{u} e^{-\left\langle\cdot, V_{u} f\right\rangle}\left(Y_{t}\right) \mid \mathscr{F}_{s}\right] \\
& =\mathbf{E}_{\mu}^{Y}\left[\partial_{u=0+} \mathbf{T}_{u} e^{-\left\langle\cdot, V_{u} f\right\rangle}\left(Y_{t}\right) \mid \mathscr{F}_{s}\right] \\
& =\mathbf{E}_{\mu}^{Y}\left[\mathscr{L}^{Y} e^{-\langle\cdot, f\rangle}\left(Y_{t}\right) \mid \mathscr{F}_{s}\right] .
\end{aligned}
$$

By using the above results it is not difficult to prove the semi-martingale representation of $Y_{t}$ as of $X_{t}$ in [2]. In fact, for $f \in C_{c}^{\infty},\left\langle Y_{t}, f\right\rangle$ is a special semimartingale, thus,

$$
\left\langle Y_{t}, f\right\rangle=\left\langle Y_{0}, f\right\rangle+C_{t}(f)+M_{t}^{c}(f)+\tilde{N}_{t}(f)+N_{t}(f),
$$


where $C_{t}(f)$ is a continuous process of locally bounded variation, $M_{t}^{c}(f)$ is a continuous $L^{2}$-martingale with quadratic variation $\left\langle\left\langle M^{c}(f)\right\rangle\right\rangle_{t}$, and

$$
\begin{aligned}
& \tilde{N}_{t}(f)=\int_{0}^{t} \int_{\mathscr{M}^{ \pm}}\langle\mu, f\rangle I(\|\mu\|<1) \tilde{N}(d s, d \mu), \\
& N_{t}(f)=\int_{0}^{t} \int_{\mathscr{M}^{ \pm}}\langle\mu, f\rangle I(\|\mu\| \geq 1) N(d s, d \mu)
\end{aligned}
$$

with the jump measure $N$ of $\left\{Y_{t}\right\}$, its compensator $\hat{N}$ and $\tilde{N}=N-\hat{N}$.

If we set

$$
\begin{aligned}
B_{t}(f)= & C_{t}(f)+\int_{0}^{t} \int_{\{\|\mu\| \geq 1\}}\langle\mu, f\rangle \hat{N}(d s, d \mu) \\
& +\lambda \int_{0}^{t} d s \int\left\langle\delta_{w_{n}(s)}-\delta_{w_{m}(s)}, f\right\rangle q(d(m, n)),
\end{aligned}
$$

then by applying Ito's formula for $Z_{t}(f)$ we can get

$$
\begin{aligned}
& -d B_{t}(f)+\frac{1}{2} d\left\langle\left\langle M^{c}(f)\right\rangle\right\rangle_{t}+\int\left[e^{-\langle\mu, f\rangle}-1+\langle\mu, f\rangle\right] \hat{N}(d t, d \mu) \\
& =-\Psi\left(Y_{t} ; f\right) d t \\
& =\left\{-\left\langle Y_{t}, A f\right\rangle+\left\langle Y_{t}, \Gamma f\right\rangle+\lambda \int\left(e^{-\left\langle\delta_{w_{n}^{*}(t)}-\delta_{w_{m}^{*}(t)}, f\right\rangle}-1\right) q(d(m, n))\right\} d t \\
& =\left\{-\left[\left\langle Y_{t}, A f\right\rangle+\lambda \int\left\langle\delta_{w_{n}^{*}(t)}-\delta_{w_{m}^{*}(t)}, f\right\rangle q(d(m, n))\right]+\left\langle Y_{t}, \Gamma^{c} f\right\rangle\right. \\
& \left.+\left\langle Y_{t}, \Gamma^{d} f\right\rangle+\lambda \int\left[e^{-\left\langle\delta_{w_{n}^{*}(t)}-\delta_{w_{m}^{*}(t)}, f\right\rangle}-1+\left\langle\delta_{w_{n}^{*}(t)}-\delta_{w_{m}^{*}(t)}, f\right\rangle\right] q(d(m, n))\right\} d t
\end{aligned}
$$

Thus we have

$$
\begin{aligned}
B_{t}(f) & =\int_{0}^{t}\left\langle Y_{s}, A f\right\rangle d s+\lambda \int\left\langle\delta_{w_{n}^{*}(t)}-\delta_{w_{m}^{*}(t)}, f\right\rangle q(d(m, n)), \\
\left\langle\left\langle M^{c}(f)\right\rangle\right\rangle_{t} & =2 \int_{0}^{t}\left\langle Y_{s}, \Gamma^{c} f\right\rangle d s=\int_{0}^{t}\left\langle Y_{s}, Q^{c} f\right\rangle d s
\end{aligned}
$$

and 


$$
\begin{aligned}
\hat{N}(d s, d \mu)=d s\{ & \int Y_{s}(d x)\left(\int v(x, d y) \delta_{\left(\delta_{y}-\delta_{x}\right)}+k(x) \delta_{-\delta_{x}}\right) \\
& \left.+\lambda \int q(d(m, n)) \delta_{\left(\delta_{w_{n}^{*}(s)}-\delta_{w_{m}^{*}(s)}\right)}\right\}(d \mu) .
\end{aligned}
$$

Therefore the proof is completed.

\section{Martingale Problems for $\mathscr{L}^{Y}$}

The following assumption is needed to prove the well-posedness of martingale problems.

Assumption 3. For each $f \in\left(C_{c}^{\infty}\right)^{+}, A V_{t} f=-A \log \left(1-P_{t}\left(1-e^{-f}\right)\right)$ is well-defined and $A V_{t} f$ is continuous in $t$ under the norm $\left\|\cdot / g_{1}\right\|_{\infty}$, i.e.,

$$
\left\|\left(A V_{t} f-A V_{t_{0}} f\right) / g_{1}\right\|_{\infty} \rightarrow 0 \quad\left(t \rightarrow t_{0}\right) .
$$

In the following we suppose that the generator $A$ of the motion process has the form of (1.1).

For $\eta \in \mathscr{M}_{g_{0}}$, let $F(\eta)=\Phi\left(\left\langle\eta, f_{1}\right\rangle, \ldots,\left\langle\eta, f_{n}\right\rangle\right) \in \mathscr{D}_{0} \stackrel{\text { def }}{\Longleftrightarrow} \Phi(x) \in C^{\infty}\left(\mathbf{R}^{n}\right)$ is a polynomial growth function with polynomial growth derivatives of all orders and $f_{i} \in D_{g}, i=1, \ldots, n$. For this $F(\eta)$, the generator $\mathscr{L}_{0}$ of $X_{t}$ will be extended to the following form:

$$
\begin{aligned}
\mathscr{L}_{0} F(\eta)= & \sum_{i=1}^{n} \partial_{i} \Phi\left(\left\langle\eta, f_{1}\right\rangle, \ldots,\left\langle\eta, f_{n}\right\rangle\right)\left\langle\eta, A f_{i}\right\rangle \\
& +\frac{1}{2} \sum_{i, j=1}^{n} \partial_{i j}^{2} \Phi\left(\left\langle\eta, f_{1}\right\rangle, \ldots,\left\langle\eta, f_{n}\right\rangle\right)\left\langle\eta, Q^{c}\left(f_{i}, f_{j}\right)\right\rangle \\
& +\int_{S}\left\{\int _ { S \backslash \{ x \} } v ( x , d y ) \left[\begin{array}{r}
\Phi\left(\left\langle\eta, f_{1}\right\rangle+f_{1}(y)-f_{1}(x), \ldots,\left\langle\eta, f_{n}\right\rangle\right. \\
\left.+f_{n}(y)-f_{n}(x)\right)-\Phi\left(\left\langle\eta, f_{1}\right\rangle, \ldots,\left\langle\eta, f_{n}\right\rangle\right) \\
\left.-\sum_{i=1}^{n} \partial_{i} \Phi\left(\left\langle\eta, f_{1}\right\rangle, \ldots,\left\langle\eta, f_{n}\right\rangle\right)\left(f_{i}(y)-f_{i}(x)\right)\right]
\end{array}\right.\right.
\end{aligned}
$$




$$
\begin{aligned}
+k(x)[ & \Phi\left(\left\langle\eta, f_{1}\right\rangle-f_{1}(x), \ldots,\left\langle\eta, f_{n}\right\rangle-f_{n}(x)\right) \\
& -\Phi\left(\left\langle\eta, f_{1}\right\rangle, \ldots,\left\langle\eta, f_{n}\right\rangle\right) \\
& \left.\left.+\sum_{i=1}^{n} \partial_{i} \Phi\left(\left\langle\eta, f_{1}\right\rangle, \ldots,\left\langle\eta, f_{n}\right\rangle\right) f_{i}(x)\right]\right\} \eta(d x),
\end{aligned}
$$

where

$$
Q^{c}(f, g)(x)=\sum_{i, j} a^{i j}(x) \partial_{i} f(x) \partial_{i} g(x)
$$

For $F(\eta) \in \mathscr{D}_{0}$, the generator $\mathscr{L}^{Y}$ of $Y_{t}$ will be extended to

$$
\mathscr{L}^{Y} F(\eta)=\mathscr{L}_{0} F(\eta)+\lambda \int\left(\Theta_{m, n} F(\eta)-F(\eta)\right) q(d(m, n)) .
$$

THEOREM 4 (Martingale Problem for $\left(\mathscr{L}^{Y}, \mathscr{D}_{0}, \mu\right)$ ). Under Assumption 1, 2 and 3, suppose that the generator $A$ is given as in (1.1). Let $\mu \in \mathscr{M}_{g_{0}}$.

(i) $\mathbf{P}_{\mu}^{Y}\left(Y_{0}=\mu\right)=1$ holds and for each $F(\mu)=\Phi\left(\left\langle\mu, f_{1}\right\rangle, \ldots,\left\langle\mu, f_{n}\right\rangle\right) \in \mathscr{D}_{0}$,

$$
M_{t}^{F}=F\left(Y_{t}\right)-F\left(Y_{0}\right)-\int_{0}^{t} \mathscr{L}^{Y} F\left(Y_{s}\right) d s \text { is } \mathbf{P}_{\mu}^{Y} \text {-martingale. }
$$

(ii) If there is a probability measure $\mathbf{Q}_{\mu}$ on $\mathbf{D}=\mathbf{D}\left([0, \infty) \rightarrow \mathscr{M}_{g_{0}}\right)$ such that the canonical process $\tilde{Y}_{t}(\omega)=\omega(t) \quad(\omega \in \mathbf{D})$ satisfies the same conditions as $\left(Y_{t}, \mathbf{P}_{\mu}^{Y}\right)$ in (i) and

$$
\int_{0}^{t}\left\langle\tilde{Y}_{s}, g_{1}\right\rangle d s<\infty \quad \mathbf{Q}_{\mu} \text {-a.s. for all } t \geq 0,
$$

then $\mathbf{Q}_{\mu}=\mathbf{P}_{\mu}^{Y} \circ Y^{-1}$ on $\mathbf{D}$, that is, martingale problem for $\left(\mathscr{L}^{Y}, \mathscr{D}_{0}, \mu\right)$ on $\mathbf{D}$ is well-posed.

Proof. The proof is essentially the same as the independent case (see $\S 5$ in [2]). However, the computations are more complicated, so we give the outline of the proof. (i) is easily obtained. We show (ii). We always fix $f \in C_{c}^{\infty}, T>0$, and set $\Psi_{t}^{T}(\eta)=\mathbf{T}_{T-t}\left(\exp -\left\langle\cdot, V_{T-t} f\right\rangle\right)(\eta)\left(0 \leq t \leq T, \eta \in \mathscr{M}_{g_{0}}\right)$. It is no difficult to show that $\left\{\Psi_{t}^{T}\left(\tilde{Y}_{t}\right)\right\}_{t \leq T}$ is a $\mathbf{Q}_{\mu}$-martingale. In fact, by using Ito's formula 


$$
\begin{aligned}
d\left(\exp -\left\langle\tilde{Y}_{t}, f\right\rangle\right)= & -\left\langle\tilde{Y}_{t}, A f-\Gamma f\right\rangle e^{-\left\langle\tilde{Y}_{t}, f\right\rangle} d t \\
& +\lambda \int q(d(m, n))\left(\Theta_{m, n}-I\right) e^{-\langle\cdot, f\rangle}\left(\tilde{Y}_{t}\right) d t+d\left(\mathbf{Q}_{\mu} \text {-martingale }\right) .
\end{aligned}
$$

Since $\mathbf{T}_{t}$ is a bounded operator, we have (set $v_{t}=v_{t}^{T}=V_{T-t} f$ again)

$$
\begin{aligned}
d\left(\Psi_{t}^{T}\left(\tilde{Y}_{t}\right)\right)= & \mathbf{T}_{T-t}\left(-\lambda \int q(d(m, n))\left(\Theta_{m, n}-I\right) e^{-\left\langle\cdot, v_{t}\right\rangle}-\left\langle\cdot, \partial_{t} v_{t}\right\rangle e^{-\left\langle\cdot, v_{t}\right\rangle}\right. \\
& \left.-\left\langle\cdot, A v_{t}-\Gamma v_{t}\right\rangle e^{-\left\langle\cdot, v_{t}\right\rangle}+\lambda \int q(d(m, n))\left(\Theta_{m, n}-I\right) e^{-\left\langle\cdot, v_{t}\right\rangle}\right)\left(\tilde{Y}_{t}\right) d t \\
& +d\left(\mathbf{Q}_{\mu} \text {-martingale }\right) \\
= & \mathbf{T}_{T-t}\left(-\left\langle\cdot, \partial_{t} v_{t}+A v_{t}-\Gamma v_{t}\right\rangle e^{-\left\langle\cdot, v_{t}\right\rangle}\right)\left(\tilde{Y}_{t}\right)+d\left(\mathbf{Q}_{\mu} \text {-martingale }\right) \\
= & d\left(\mathbf{Q}_{\mu} \text {-martingale }\right)
\end{aligned}
$$

Hence for $0 \leq s<t \leq T$, we have

$$
\mathbf{Q}_{\mu}\left[\Psi_{t}^{T}\left(\tilde{Y}_{t}\right) \mid \tilde{F}_{s}\right]=\Psi_{s}^{T}\left(\tilde{Y}_{s}\right)
$$

and set $T=t$, then

$$
\mathbf{Q}_{\mu}\left[e^{-\left\langle\tilde{Y}_{t}, f\right\rangle} \mid \mathscr{F}_{s}\right]=\mathbf{T}_{t-s} e^{-\left\langle\cdot, V_{t-s} f\right\rangle}\left(\tilde{Y}_{s}\right) .
$$

Therefore $\mathbf{P}_{\mu}=\mathbf{Q}_{\mu}$ on $\mathbf{D}$.

\section{Multi-Dimensional Absorbing Stable Motions on a Half Space}

In $\S 3$ of [2] as a motion process we considered absorbing Brownian motion and absorbing stable motion on $(0, \infty)$ and discussed the Hölder (right) continuities of $\left\{X_{t}\right\}$. It is possible to consider absorbing motions on $H=\mathbf{R}^{d-1} \times$ $(0, \infty)$ and we can get the same results as in Theorem 3.1 and in Corollary 3.1 of [2]. For the absorbing Brownian motion, it is not so difficult and essentially done in [1]. So in this section we only discuss the absorbing stable motion on $H$.

For a function $f$ on $H$, let $\bar{f}$ be an extension of $f$ to on $\mathbf{R}^{d}$ defined as

$$
\bar{f}(x)= \begin{cases}f(x) & \left(x_{d}>0\right) \\ f(\tilde{x}, 0+)=0 & \left(x_{d}=0\right) \\ -f\left(\tilde{x},-x_{d}\right) & \left(x_{d}<0\right)\end{cases}
$$


where $x=\left(\tilde{x}, x_{d}\right) \in H$. Note that if $x \in H$, then $\bar{f}(x)=f(x)$. The generator $A^{-} \equiv A^{-, \alpha}$ of absorbing $\alpha$-stable motion $\left(w^{-}(t), P_{x}^{-}\right) \equiv\left(w^{-, \alpha}(t), P_{x}^{-, \alpha}\right)$ on $H$ is given as $A^{-, \alpha} f(x)=A^{\alpha} \bar{f}(x) ;\left(A^{-}\right.$is the same as $L^{-}$in $\S 4$ of [1], however, in which we have some miss-prints)

$$
\begin{aligned}
A^{-, \alpha} f(x)= & c \int_{\mathbf{R}^{d} \backslash\{0\}}[\bar{f}(x+y)-\bar{f}(x)-\nabla \bar{f}(x) \cdot y I(|y|<1)] \frac{d y}{|y|^{d+\alpha}} \\
= & c \int_{\mathbf{R}^{d-1}} d \tilde{y} \int_{-x_{d}}^{x_{d}}[f(x+y)-f(x)-\nabla f(x) \cdot y I(|y|<1)] \frac{d y_{d}}{|y|^{d+\alpha}} \\
& +c \int_{\mathbf{R}^{d-1}} d \tilde{y} \int_{x_{d}}^{\infty}\left[f(x+y)-f\left(\tilde{x}+\tilde{y}, y_{d}-x_{d}\right)-2 f(x)\right] \frac{d y_{d}}{|y|^{d+\alpha}}
\end{aligned}
$$

with some positive constant $c$, where in the last term the integral corresponding to $\nabla f(x) \cdot y$ is equal to zero by the symmetric property (of course, it is integrable).

We can also write that if $0<\alpha<1$, then

$$
\begin{aligned}
A^{-, \alpha} f(x)=c \int_{\mathbf{R}^{d} \backslash\{x\}}[\bar{f}(y)-\bar{f}(x)] \frac{d y}{|y-x|^{d+\alpha}} \\
=c \int_{\mathbf{R}^{d-1}} d \tilde{y}\left\{\int_{0}^{\infty}[f(y)-f(x)] K(x, y) d y_{d}\right. \\
\left.\quad-2 f(x) \int_{0}^{\infty} \frac{d y_{d}}{\left|\left(\tilde{y}-\tilde{x}, y_{d}+x_{d}\right)\right|^{d+\alpha}}\right\}, \\
=c \int_{\mathbf{R}^{d-1}} d \tilde{y} \int_{0}^{\infty}[f(y)-f(x)] K(x, y) d y_{d}-f(x) k(x),
\end{aligned}
$$

and that if $1 \leq \alpha<2$, then

$$
\begin{gathered}
A^{-, \alpha} f(x)=c \int_{\mathbf{R}^{d} \backslash\{x\}}[\bar{f}(y)-\bar{f}(x)-\nabla \bar{f}(x) \cdot(y-x) I(|y-x|<1)] \frac{d y}{|y-x|^{d+\alpha}} \\
=c \int_{\mathbf{R}^{d-1}} d \tilde{y}\left\{\int_{0}^{\infty}[f(y)-f(x)-\nabla f(x) \cdot(y-x) I(|y-x|<1)] K(x, y) d y_{d}\right. \\
+\int_{0}^{\infty}[-2 f(x)-\nabla f(x) \cdot(y-x) I(|y-x|<1) \\
\left.\times \frac{\left.-\nabla f(x) \cdot\left(\tilde{y}-\tilde{x},-y_{d}-x_{d}\right) I\left(\left|\left(\tilde{y}-\tilde{x}, y_{d}+x_{d}\right)\right|<1\right)\right]}{\left|\left(\tilde{y}-\tilde{x}, y_{d}+x_{d}\right)\right|^{d+\alpha}}\right\}
\end{gathered}
$$




$$
\begin{aligned}
= & c \int_{\mathbf{R}^{d-1}} d \tilde{y} \int_{0}^{\infty}[f(y)-f(x)-\nabla f(x) \cdot(y-x) I(|y-x|<1)] K(x, y) d y_{d} \\
& -f(x) k(x)+\nabla f(x) \cdot c(x),
\end{aligned}
$$

where

$$
\begin{gathered}
K(x, y)=\frac{I(y \neq x)}{|y-x|^{d+\alpha}}-\frac{1}{\left|\left(\tilde{y}-\tilde{x}, y_{d}+x_{d}\right)\right|^{d+\alpha}}, \\
k(x)=k\left(x_{d}\right)=2 c \int_{\mathbf{R}^{d-1}} d \tilde{y} \int_{x_{d}}^{\infty} \frac{d y_{d}}{|y|^{d+\alpha}}
\end{gathered}
$$

and

$$
\begin{gathered}
c(x)=c \int_{\mathbf{R}^{d-1}} d \tilde{y} \int_{0}^{\infty}\left[-\left(\tilde{y}-\tilde{x},-y_{d}-x_{d}\right) I\left(\left|\left(\tilde{y}-\tilde{x}, y_{d}+x_{d}\right)\right|<1\right)\right. \\
-(y-x) I(|y-x|<1)] \frac{d y_{d}}{\left|\left(\tilde{y}-\tilde{x}, y_{d}+x_{d}\right)\right|^{d+\alpha}} \\
=c \int_{\mathbf{R}^{d-1}} d \tilde{y} \int_{0}^{\infty}\left[\left(\tilde{y}, y_{d}+x_{d}\right) I\left(\left|\left(\tilde{y}, y_{d}+x_{d}\right)\right|<1\right)\right. \\
\left.\quad-\left(\tilde{y}, y_{d}-x_{d}\right) I\left(\left|\left(\tilde{y}, y_{d}-x_{d}\right)\right|<1\right)\right] \frac{d y_{d}}{\left|\left(\tilde{y}, y_{d}+x_{d}\right)\right|^{d+\alpha}} .
\end{gathered}
$$

Let $h_{0}(v)$ be a $C^{\infty}$-function on $(0, \infty)$ such that $0<h_{0} \leq 1$ on $(0, \infty)$, $h_{0}(v)=v$ for $v \in(0,1 / 2]$ and $h_{0}(v)=1$ for $v \geq 1$. Let $d<p<d+\alpha$. Set $g_{p}(x)=$ $\left(1+|x|^{2}\right)^{-p / 2}$ and $g_{p, 0}(x):=g_{p}(x) h_{0}\left(x_{d}\right)$ for $x \in H$. Let $\left.f \in C_{p} \stackrel{\text { def }}{\Longleftrightarrow} f \in C\left(\mathbf{R}^{d}\right)\right|_{H}$;

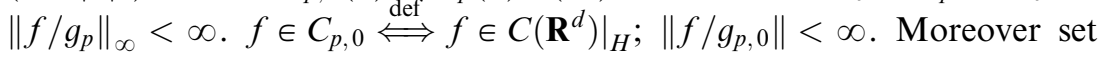

$$
\begin{aligned}
f \in C_{p, 0}^{3} & \left.\stackrel{\text { def }}{\Longleftrightarrow} f \in C_{b}^{3}\left(\mathbf{R}^{d}\right)\right|_{H} ; \\
& \text { for } i, j \neq d, f, \partial_{d}^{2} f, \partial_{i} f, \partial_{i j}^{2} f \in C_{p, 0} \text { and } \partial_{d} f, \partial_{i d}^{2} f \in C_{p} .
\end{aligned}
$$

Then we can take $D_{g}=C_{p, 0}^{3}$.

Moreover for each $0<\alpha<2, Q^{-} f \equiv Q^{-, \alpha} f=A f^{2}-f A f$ is given by the following formula:

$$
\begin{aligned}
Q^{-, \alpha} f(x)= & c \int_{\mathbf{R}^{d-1}} d \tilde{y} \int_{-x_{d}}^{x_{d}}[f(x+y)-f(x)]^{2} \frac{d y_{d}}{|y|^{d+\alpha}} \\
& +c \int_{\mathbf{R}^{d-1}} d \tilde{y} \int_{x_{d}}^{\infty}\left[\left\{f(x+y)-f\left(\tilde{y}+\tilde{x}, y_{d}-x_{d}\right)\right\}\right.
\end{aligned}
$$




$$
\begin{aligned}
& \left.\left\{f(x+y)+f\left(\tilde{y}+\tilde{x}, y_{d}-x_{d}\right)-2 f(x)\right\}+2 f(x)^{2}\right] \frac{d y_{d}}{|y|^{d+\alpha}} \\
= & c \int_{\mathbf{R}^{d-1}} d \tilde{y} \int_{0}^{\infty}[f(y)-f(x)] K(x, y) d y_{d}+f(x)^{2} k(x) .
\end{aligned}
$$

THEOREM 5. Let $d \geq 1, d<p<d+\alpha, \mu \in \mathscr{M}_{g_{p, 0}}$ and let the motion process be absorbing $\alpha$-stable motion on $H$ with $0<\alpha<2$. Let $\varepsilon>0$ denote an arbitrary small number.

(i) For $\left(X_{t}, \mathbf{P}_{\mu}\right)$ the following holds.

(a) Under $\mathbf{P}_{\mu},\left\{\left\langle X_{t}, g_{p, 0}\right\rangle\right\}$ is $(1 /(2(\alpha \vee 1))-\varepsilon)$-Hölder right continuous at $t=0$. Moreover in case of $1<\alpha<2$, if $\left\langle\mu, g_{1}\right\rangle<\infty$ with $g_{1}(x)=$ $g_{p}(x) h_{0}\left(x_{d}\right)^{2-\alpha}$, then $\left\{\left\langle X_{t}, g_{p, 0}\right\rangle\right\}$ is $(1 / 2-\varepsilon)$-Hölder right continuous at $t=0$.

(b) If $t_{0}>0$, then under $\mathbf{P}_{\mu},\left\{\left\langle X_{t}, g_{p, 0}\right\rangle\right\}$ is $(1 / 2-\varepsilon)$-Hölder right continuous at $t=t_{0}$ for every $0<\alpha<2$.

(ii) For $\left(Y_{t}, \mathbf{P}_{\mu}^{Y}\right)$ the same results hold as above.

Proof. Let $d \geq 2$. The proof is proceeded in the same way as the case of $d=1$. It suffices to check that the conditions in Assumption 1 and 2 are fulfilled with $g_{0}=g_{p, 0}$ and with suitable $g_{1} \in C^{\infty}, 0 \leq \gamma<1$ as follows. Let $h_{1} \in C^{\infty} ; 0<h_{1} \leq 1, h_{1}(v)=v \log (1 / v)$ for $v \in(0,1 / e]$ and $h_{1}(v)=1$ for $v \geq 1$.

(i) If $0<\alpha<1$, then $g_{1}(x)=g_{p, 0}(x), \gamma=0$.

(ii) If $\alpha=1$, then $g_{1}(x)=g_{p}(x) h_{1}\left(x_{d}\right), \gamma=\delta$ for any small $0<\delta<1$.

(iii) If $1<\alpha<2$, then $g_{1}(x)=g_{p}(x) h_{0}\left(x_{d}\right)^{2-\alpha}, \gamma=1-1 / \alpha$.

Note that as $x_{d} \downarrow 0$,

$$
g_{1}(x) \sim x_{d}(0<\alpha<1), \quad \sim x_{d} \log \left(1 / x_{d}\right)(\alpha=1), \quad \sim x_{d}^{2-\alpha}(1<\alpha<2) .
$$

For simplicity of the notations we omit the superscript " $\alpha$ " as $P_{t}^{-, \alpha}=P_{t}^{-}$, $A^{-, \alpha}=A^{-}$. We shall show the following. Since they imply $\left\|g_{p, 0} P_{t}^{-} g_{1}\right\|_{\infty} \leq C t^{-\gamma}$, we can get the $((1-\gamma) / 2-\epsilon)$-Hölder right continuity.

(C1) $C_{p, 0}^{3} \subset \mathscr{D}\left(A^{-}\right), P_{t}^{-} C_{c}^{\infty} \subset C_{p, 0}^{3}$ for every $t \geq 0$, $\sup _{t \geq 0,0<x_{d} \leq 1}\left|x_{d}^{-1} P_{t}^{-} g_{p, 0}(x)\right|$ $<\infty$ and $A^{-} C_{p, 0}^{3} \subset C_{p, 0}$ (these imply Assumption 1 and that $C_{p, 0}^{3}$ is a core). 
(C2) For every $f \in C_{p, 0}^{3}, \quad \partial_{t} P_{t}^{-} f^{2}(x)=A^{-} P_{t}^{-} f^{2}(x)=P_{t}^{-} A^{-} f^{2}(x) \quad(x \in H)$, $A^{-} f^{2} \in C_{b}$ and $\left\|g_{1}^{-1} Q^{-} f\right\|_{\infty}<\infty$ (these imply (i) of Assumption 2).

(C3) For each $0<\beta \leq 1$, $\sup _{t \geq 0} P_{t}^{-}\left(y_{d}^{\beta}\right)(x) \leq 2(1+\beta) x_{d}^{\beta}$ for all $x \in H$ (this implies (ii) of Assumption 2).

(C4) For each $0<\beta \leq 1$, $\sup _{0<x_{d} \leq 1} x_{d}^{-1} P_{t}^{-}\left(y_{d}^{\beta}\right)(x) \leq C_{\beta} t^{-(1-\beta) / \alpha}$ with a constant $C_{\beta}>0$ depending only on $\beta$ (this implies (iii), (iv) of Assumption 2).

Note that we take $\gamma=(1-\beta) / \alpha$ in Assumption 2. More exactly, if $0<\alpha<1$, then $\beta=1$, i.e., $\gamma=0$. If $\alpha=1$, then $\beta=1-\delta$ for any small $0<\delta<1$, i.e., $\gamma=\delta$. If $1<\alpha<2$, then $\beta=2-\alpha$, i.e., $\gamma=1-1 / \alpha$. (C3) and (C4) can be shown in a way similar to the case of $d=1$; (B3) and (B4) in [2] by using the following. For the density $p^{\alpha}(x)$ of the rotation invariant $\alpha$-stable motion on $\mathbf{R}^{d}$ starting from $0, p_{t}^{\alpha}(x)=t^{-d / \alpha} p_{1}^{\alpha}\left(t^{-1 / \alpha} x\right)$ and $p_{1}^{\alpha}(x) \leq C\left(1 \wedge|x|^{-d-\alpha}\right)$. The transition density $p_{t}^{-}(x, y) \equiv p_{t}^{-, \alpha}(x, y)$ of absorbing $\alpha$-stable motion on $H$ is given as

$$
p_{t}^{-}(x, y)=p_{t}^{\alpha}(y-x)-p_{t}^{\alpha}\left(\tilde{y}-\tilde{x}, y_{d}+x_{d}\right)=-\int_{-x_{d}}^{x_{d}} \partial_{d} p_{t}^{\alpha}\left(\tilde{y}-\tilde{x}, y_{d}+v\right) d v .
$$

We also use the following result.

$$
\int_{H} z_{d}^{\beta-1} p_{1}^{\alpha}\left(\tilde{z}, z_{d}+u\right) d z \text { is bounded in } u \in \mathbf{R} .
$$

From these results we can get (C3), (C4).

In each $(\mathrm{C} 1),(\mathrm{C} 2)$, the claims except the last one can be shown by the same way as in $d=1$. In order to show the last claims of $(\mathrm{C} 1),(\mathrm{C} 2)$, it is enough to prove that for each $f \in C_{p, 0}^{3}$, there is a constant $C>0$ such that

$$
\left|A^{-} f(x)\right| \leq C x_{d} \text { for } 0<x_{d} \leq 1 / 2 \text { and } Q^{-} f(x) \leq C g_{1}(x) \text { for all } x \in H .
$$

Let $0<x_{d} \leq 1 / 2$. For $A^{-}$we use the formula (4.1). In the following we decompose as $A^{-} f=\left(J_{1,1}+J_{1,2}\right)+\left(J_{2}+J_{3}\right)$ and we shall show each term has order of $x_{d}^{2}, x_{d}, x_{d}, x_{d}$, respectively. The main calculus is of $J_{1,2}(1<\alpha<2)$ and $J_{3}$. In the first term of (4.1) we divide the integral area to $\{|y| \geq 1\},\{|y|<1\}$ and denote the corresponding terms by $J_{1,1}(x), J_{1,2}(x)$ respectively. In the following we use the same symbols $C^{\prime}, C^{\prime \prime}$ as any positive finite constants which are independent of $x$. First note that if $|y| \geq 1$ and $\left|y_{d}\right| \leq x_{d} \leq 1 / 2$, then $|\tilde{y}|^{2} \geq 1-x_{d}^{2} \geq 3 / 4=: b$. By $|f(x)| \leq C x_{d}$, 


$$
\begin{aligned}
\left|J_{1,1}(x)\right| & =\left|c \int_{\mathbf{R}^{d-1}} d \tilde{y} \int_{-x_{d}}^{x_{d}}[f(x+y)-f(x)] I(|y| \geq 1) \frac{d y_{d}}{|y|^{d+\alpha}}\right| \\
& \leq 2 c \int_{|\tilde{y}| \geq b} d \tilde{y} \int_{0}^{x_{d}} C\left(2 x_{d}+y_{d}\right) \frac{d y_{d}}{|y|^{d+\alpha}} \\
& \leq 2 c C \int_{|\tilde{y}| \geq b} \frac{d \tilde{y}}{|\tilde{y}|^{d+\alpha}} \int_{0}^{x_{d}}\left(2 x_{d}+y_{d}\right) d y_{d} \\
& \leq 2 c C \int_{b}^{\infty} \frac{d r}{r^{2+\alpha}} \int_{0}^{x_{d}}\left(2 x_{d}+y_{d}\right) d y_{d} \\
& =\frac{5 c C}{1+\alpha} b^{-1-\alpha} x_{d}^{2}=C^{\prime} x_{d}^{2} .
\end{aligned}
$$

Next note that if $|y|<1$, then $|\tilde{y}|<1$ and that for some $\theta \in(0,1)$,

$$
|f(x+y)-f(x)-\nabla f(x) \cdot y|=\frac{1}{2}\left|f^{(2)}(x+\theta y) \cdot y^{2}\right| \leq \frac{1}{2}\left\|f^{(2)}\right\|_{\infty}|y|^{2} .
$$

If $0<\alpha<1$, then $|y|^{d-2+\alpha} \geq|\tilde{y}|^{d-2+\alpha}$ by $d-2+\alpha>0$, and

$$
\begin{aligned}
\left|J_{1,2}(x)\right| & =\left|c \int_{\mathbf{R}^{d-1}} d \tilde{y} \int_{-x_{d}}^{x_{d}}[f(x+y)-f(x)-\nabla f(x) \cdot y] I(|y|<1) \frac{d y_{d}}{|y|^{d+\alpha} \mid}\right| \\
& \leq c \int_{|\tilde{y}|<1} d \tilde{y} \int_{-x_{d}}^{x_{d}}|f(x+y)-f(x)-\nabla f(x) \cdot y| \frac{d y_{d}}{|y|^{d+\alpha}} \\
& \leq c \int_{|\tilde{y}|<1} d \tilde{y} \int_{0}^{x_{d}}\left\|f^{(2)}\right\|_{\infty}|y|^{2} \frac{d y_{d}}{|y|^{d+\alpha}} \\
& \leq c \int_{|\tilde{y}|<1} \frac{d \tilde{y}}{|\tilde{y}|^{d+\alpha-2}}\left\|f^{(2)}\right\|_{\infty} x_{d} \\
& \leq c \int_{0}^{1} r^{-\alpha} d r\left\|^{(2)}\right\|_{\infty} x_{d} \\
& =\frac{c\left\|f^{(2)}\right\| \|_{\infty}}{1-\alpha} x_{d}=C^{\prime} x_{d} .
\end{aligned}
$$

On the other hand if $1 \leq \alpha<2$, then by using

$$
f(x+y)-f(x)-\nabla f(x) \cdot y=\frac{1}{2} f^{(2)}(x) \cdot y^{2}+\frac{1}{6} f^{(3)}(x+\theta y) \cdot y^{3}
$$


with some $\theta \in(0,1)$, and the corresponding integral to $\sum_{i=1}^{d-1} \partial_{i d}^{2} f(x) y_{i} y_{d}$ is equal to zero by symmetric property in $y_{d}$, we have

$$
\begin{aligned}
\left|J_{1,2}(x)\right| \leq c \int_{|\tilde{y}|<1} d \tilde{y} \int_{0}^{x_{d}}\left\{\sum_{i, j=1}^{d-1}\left|\partial_{i j}^{2} f(x)\right|\left|y_{i}\right|\left|y_{j}\right|+\left|\partial_{d}^{2} f(x)\right| y_{d}^{2}\right. \\
\left.+\frac{1}{3}\left\|f^{(3)}\right\||y|^{3}\right\} \frac{d y_{d}}{|y|^{d+\alpha}} .
\end{aligned}
$$

Let $0<\epsilon<2-\alpha$ and set $\alpha_{\epsilon}:=\alpha+\epsilon \in(1,2)$, then $|y|^{d+\alpha-2} \geq|\tilde{y}|^{d-1-\epsilon}\left|y_{d}\right|^{-1+\alpha_{\epsilon}}$. By $\left|\partial_{i j}^{2} f(x)\right|,\left|\partial_{d}^{2} f(x)\right| \leq C x_{d}$ for $i, j \neq d$, corresponding terms to $f^{(2)}$ are less than or equal to

$$
C x_{d} \int_{|\tilde{y}|<1} \frac{d \tilde{y}}{|\tilde{y}|^{d-1-\epsilon}} \int_{0}^{x_{d}} \frac{d y_{d}}{y_{d}^{-1+\alpha_{\epsilon}}}=C x_{d} \int_{0}^{1} r^{\epsilon-1} d r \frac{x_{d}^{2-\alpha_{\epsilon}}}{2-\alpha_{\epsilon}}=\frac{C}{\left(2-\alpha_{\epsilon}\right) \epsilon} x_{d}^{3-\alpha_{\epsilon}} .
$$

For the last term, by $d \geq 2, \alpha \geq 1$, i.e., $d+\alpha-3 \geq 0$, we have $|y|^{d+\alpha-3} \geq$ $|\tilde{y}|^{d+\alpha-3}$. Hence the last term is less than or equal to

$$
\int_{|\tilde{y}|<1} \frac{d \tilde{y}}{|\tilde{y}|^{d+\alpha-3}}\left\|f^{(3)}\right\|_{\infty} x_{d}=\int_{0}^{1} r^{1-\alpha} d r\left\|f^{(3)}\right\|_{\infty} x_{d}=\frac{\left\|f^{(3)}\right\|_{\infty}}{2-\alpha} x_{d} .
$$

These estimates imply $\left|J_{1,2}(x)\right| \leq C^{\prime} x_{d}$. In the second term of (4.1) we also divide the integral area to $\{|y| \geq 1\},\{|y|<1\}$ and denote the corresponding terms by $J_{2}(x), J_{3}(x)$ respectively. For $J_{2}(x)$, by

$$
\left|f(x+y)-f\left(\tilde{y}+\tilde{x}, y_{d}-x_{d}\right)\right| \leq 2 x_{d}\left\|\partial_{d} f\right\|_{\infty}
$$

and $|f(x)| \leq C x_{d}$, we have

$$
\begin{aligned}
\left|J_{2}(x)\right| & =\left|c \int_{\mathbf{R}^{d-1}} d \tilde{y} \int_{x_{d}}^{\infty}\left[f(x+y)-f\left(\tilde{x}+\tilde{y}, y_{d}-x_{d}\right)-2 f(x)\right] I(|y| \geq 1) \frac{d y_{d}}{|y|^{d+\alpha}}\right| \\
& \leq c \int_{H} 2\left(\left\|\partial_{d} f\right\|_{\infty}+C\right) x_{d} I(|y| \geq 1) \frac{d y}{|y|^{d+\alpha}} \\
& \leq C^{\prime} x_{d} \int_{|y| \geq 1} \frac{d y}{|y|^{d+\alpha}}=C^{\prime \prime} x_{d} .
\end{aligned}
$$

For $J_{3}(x)=c \int_{\mathbf{R}^{d-1}} d \tilde{y} \int_{x_{d}}^{\infty}\left[f(x+y)-f\left(\tilde{y}+\tilde{x}, y_{d}-x_{d}\right)-2 f(x)\right] I(|y|<1) \frac{d y_{d}}{|y|^{d+\alpha}}$,

$$
\begin{aligned}
& f(x+y)-f\left(\tilde{x}+\tilde{y}, y_{d}-x_{d}\right)-2 f(x) \\
& \quad=\left[f(x+y)-f\left(\tilde{x}+\tilde{y}, y_{d}-x_{d}\right)-2 f\left(\tilde{x}+\tilde{y}, x_{d}\right)\right]-2\left[f\left(\tilde{x}+\tilde{y}, x_{d}\right)-f(x)\right] .
\end{aligned}
$$


For the first term, by the same way as in case of $d=1$ (in $\left.J_{3}(x)\right)$ corresponding to the variable $y_{d}$, we have

$$
\begin{aligned}
& \left|f(x+y)-f\left(\tilde{x}+\tilde{y}, y_{d}-x_{d}\right)-2 f(x)\right| \\
& \quad \leq 2\left\|\partial_{d}^{3} f\right\|_{\infty} x_{d}\left(y_{d}^{2}+x_{d}^{2}\right)+C\left(y_{d}+x_{d}\right) x_{d} y_{d}+2 C x_{d}^{3} .
\end{aligned}
$$

For the second term, note that

$$
f\left(\tilde{x}+\tilde{y}, x_{d}\right)-f(x)=\sum_{i=1}^{d-1} \partial_{i} f\left(\tilde{x}, x_{d}\right) y_{i}+\frac{1}{2} \sum_{i, j=1}^{d-1} \partial_{i j}^{2} f\left(\tilde{x}+\theta \tilde{y}, x_{d}\right) y_{i} y_{j}
$$

and $\left|\partial_{i j}^{2} f\left(\tilde{x}+\theta \tilde{y}, x_{d}\right)\right| \leq C x_{d}$ for $i, j \leq d$. Moreover note that by the symmetric property in $y_{i}$ we have

$$
\begin{array}{rl}
\int_{\mathbf{R}^{d-1}} & d \tilde{y} \int_{x_{d}}^{\infty} \sum_{i=1}^{d-1} \partial_{i} f\left(\tilde{x}, x_{d}\right) y_{i} I(|y|<1) \frac{d y_{d}}{|y|^{d+\alpha}} \\
= & \sum_{i=1}^{d-1} \partial_{i} f\left(\tilde{x}, x_{d}\right) \int_{x_{d}}^{1} d y_{d} \int_{|\tilde{y}|<\sqrt{1-y_{d}^{2}}} \frac{y_{i} d \tilde{y}}{|y|^{d+\alpha}}=0 .
\end{array}
$$

Let $0<\epsilon<2-\alpha$ and set $\alpha_{\epsilon}:=\alpha+\epsilon \in(0,2)$, then $|y|^{d+\alpha} \geq|\tilde{y}|^{d-1-\epsilon}\left|y_{d}\right|^{1+\alpha_{\epsilon}}$ by $d \geq 2$. Thus we can get the following: by $x_{d} \leq y_{d}$,

$$
\begin{aligned}
\left|J_{3}(x)\right| \leq & c \int_{|\tilde{y}|<1} d \tilde{y} \int_{x_{d}}^{1}\left[2\left\|\partial_{d}^{3} f\right\|_{\infty} x_{d}\left(y_{d}^{2}+x_{d}^{2}\right)+C\left(y_{d}+x_{d}\right) x_{d} y_{d}+2 C x_{d}^{3}\right] \frac{d y_{d}}{|y|^{d+\alpha}} \\
& +c \int_{|\tilde{y}|<1} d \tilde{y} \int_{x_{d}}^{1} \frac{1}{2} \sum_{i, j=1}^{d-1}\left|\partial_{i j}^{2} f\left(\tilde{x}+\theta \tilde{y}, x_{d}\right) y_{i} y_{j}\right| \frac{d y_{d}}{y_{d}^{d+\alpha}} \\
\leq & C^{\prime} x_{d} \int_{|\tilde{y}|<1} \frac{d \tilde{y}}{|\tilde{y}|^{d-1-\epsilon}} \int_{x_{d}}^{1}\left(y_{d}^{2}+x_{d} y_{d}+x_{d}^{2}\right) \frac{d y_{d}}{y_{d}^{1+\alpha_{\epsilon}}} \\
& +c \int_{|\tilde{y}|<1} \frac{1}{2} C x_{d}|\tilde{y}|^{2} d \tilde{y} \int_{0}^{1} \frac{d y_{d}}{|y|^{d+\alpha}} \\
\leq & 3 C^{\prime} x_{d} \frac{1}{\varepsilon} \int_{0}^{1} y_{d}^{1-\alpha_{\varepsilon}} d y_{d}+\frac{c C}{2} x_{d} \int_{|\tilde{y}|<1} d \tilde{y}|\tilde{y}|^{2} \int_{0}^{1} \frac{d y_{d}}{|y|^{d+\alpha}}
\end{aligned}
$$

For the second term if $0<\alpha<1$, then by $|y|^{d+\alpha} \geq|\tilde{y}|^{d} y_{d}^{\alpha}$,

$$
\int_{|\tilde{y}|<1} d \tilde{y}|\tilde{y}|^{2} \int_{0}^{1} \frac{d y_{d}}{|y|^{d+\alpha}} \leq \int_{|\tilde{y}|<1} \frac{|\tilde{y}|^{2}}{|\tilde{y}|^{d}} d \tilde{y} \int_{0}^{1} \frac{d y_{d}}{y_{d}^{\alpha}}=\frac{1}{1-\alpha}
$$


or if $1 \leq \alpha<2$, then by $|y|^{d+\alpha} \geq|\tilde{y}|^{d+1-\epsilon} y_{d}^{\alpha_{\epsilon}-1}$,

$$
\int_{|\tilde{y}|<1} d \tilde{y}|\tilde{y}|^{2} \int_{0}^{1} \frac{d y_{d}}{|y|^{d+\alpha}} \leq \int_{|\tilde{y}|<1} \frac{|\tilde{y}|^{2}}{|\tilde{y}|^{d+1-\epsilon}} d \tilde{y} \int_{0}^{1} \frac{d y_{d}}{y_{d}^{\alpha_{\epsilon}-1}}=\frac{1}{\epsilon\left(2-\alpha_{\epsilon}\right)} .
$$

Therefore

$$
\begin{aligned}
\left|J_{3}(x)\right| & \leq \begin{cases}\left(\frac{3 C^{\prime}}{\epsilon\left(2-\alpha_{\epsilon}\right)}+\frac{c C}{2(1-\alpha)}\right) x_{d} & (0<\alpha<1) \\
\frac{6 C^{\prime}+c C}{2 \epsilon\left(2-\alpha_{\epsilon}\right)} x_{d} & (1 \leq \alpha<2)\end{cases} \\
& =C^{\prime \prime} x_{d}
\end{aligned}
$$

Therefore we have $\left|A^{-} f(x)\right| \leq C^{\prime \prime} x_{d}$.

Next in order to show $Q^{-} f(x) \leq C g_{1}(x)$, it suffices to prove that there is a constant $C>0$ such that for $0<x_{d} \leq 1$, if $0<\alpha<1$, then $Q^{-} f(x) \leq C x_{d}$, if $\alpha=1$, then $Q^{-} f(x) \leq C x_{d} \log \left(1 / x_{d}\right)$ if $1<\alpha<2$, then $Q^{-} f(x) \leq C x_{d}^{2-\alpha}$. We use the first formula of (4.2). In the following we decompose as $Q^{-} f=\left(R_{1}+R_{2}\right)+$ $\left(S_{1}+S_{2}\right)$ and we shall show each $R_{1}, R_{2}, S_{1}$ has order of $x_{d}^{3}, x_{d}^{2-\alpha}, x_{d}$ respectively, and the main parts is $S_{2}$. In the first term of the right hand side of (4.2), we divide the integral area of $\mathbf{R}^{d-1}$ to $\{|\tilde{y}| \geq 1\},\{|\tilde{y}|<1\}$ and denote the corresponding terms by $R_{1}(x), R_{2}(x)$ respectively. By $f(x) \leq C x_{d}$, we have

$$
\begin{aligned}
R_{1}(x) & =c \int_{|\tilde{y}| \geq 1} d \tilde{y} \int_{-x_{d}}^{x_{d}}[f(x+y)-f(x)]^{2} \frac{d y_{d}}{|y|^{d+\alpha}} \\
& \leq 2 c \int_{|\tilde{y}| \geq 1} \frac{d \tilde{y}}{|\tilde{y}|^{d+\alpha}} \int_{0}^{x_{d}} C^{2}\left(2 x_{d}+y_{d}\right)^{2} d y_{d} \\
& =C x_{d}^{3}
\end{aligned}
$$

For $R_{2}$, by $\left|\partial_{i} f(x)\right| \leq C x_{d}$ if $i \neq d$,

$$
\begin{aligned}
R_{2}(x) & =2 c \int_{|\tilde{y}|<1} d \tilde{y} \int_{0}^{x_{d}}[f(x+y)-f(x)]^{2} \frac{d y_{d}}{|y|^{d+\alpha}} \\
& \leq 2 c \int_{|\tilde{y}|<1} d \tilde{y} \int_{0}^{x_{d}}\left[C\left(x_{d}+y_{d}\right) \sum_{i=1}^{d-1} y_{i}+\left\|\partial_{d} f\right\|_{\infty} y_{d}\right]^{2} \frac{d y_{d}}{|y|^{d+\alpha}} \\
& \leq C \int_{|\tilde{y}|<1} d \tilde{y} \int_{0}^{x_{d}}\left[\left(x_{d}^{2}+y_{d}^{2}\right)|\tilde{y}|^{2}+y_{d}^{2}\right] \frac{d y_{d}}{|y|^{d+\alpha}}
\end{aligned}
$$


In the above we first consider the last term (which is the main term), i.e., $\int_{|\tilde{y}|<1} d \tilde{y} \int_{0}^{x_{d}} y_{d}^{2} \frac{d y_{d}}{|y|^{d+\alpha}}=\int_{0}^{x_{d}} d y_{d} y_{d}^{2}\left(\int_{|\tilde{y}|<y_{d}}+\int_{y_{d} \leq|\tilde{y}|<1}\right) \frac{d \tilde{y}}{|y|^{d+\alpha}}=: R_{2,1}(x)+R_{2,2}(x)$.

For $R_{2,1}$, let $\alpha_{\epsilon}=\alpha+\epsilon<2$ be the same as before. By $|y|^{d+\alpha} \geq|\tilde{y}|^{d-1-\epsilon}\left|y_{d}\right|^{1+\alpha_{\epsilon}}$,

$$
\int_{|\tilde{y}|<y_{d}} \frac{d \tilde{y}}{|\tilde{y}|^{d-1-\epsilon}}=\int_{0}^{y_{d}} r^{\epsilon-1} d r=\frac{y_{d}^{\epsilon}}{\epsilon} .
$$

Hence

$$
R_{2,1}(x) \leq \int_{0}^{x_{d}} \frac{y_{d}^{2}}{y_{d}^{1+\alpha+\epsilon}} \frac{y_{d}^{\epsilon}}{\epsilon} d y_{d}=\frac{1}{\epsilon} \int_{0}^{x_{d}} y_{d}^{1-\alpha} d y_{d}=\frac{1}{(2-\alpha) \epsilon} x_{d}^{2-\alpha}
$$

For $R_{2,2}$, by $|y|^{d+\alpha} \geq|\tilde{y}|^{d+\alpha}$ and

$$
\int_{y_{d} \leq|\tilde{y}|<1} \frac{d \tilde{y}}{|\tilde{y}|^{d+\alpha}}=\int_{y_{d}}^{1} \frac{d r}{r^{2+\alpha}}=\frac{1}{1+\alpha}\left(y_{d}^{-1-\alpha}-1\right) \leq \frac{1}{1+\alpha} y_{d}^{-1-\alpha}
$$

Hence

$$
R_{2,2}(x) \leq \int_{0}^{x_{d}} y_{d}^{2} \frac{1}{1+\alpha} y_{d}^{-1-\alpha} d y_{d}=\frac{1}{1+\alpha} \int_{0}^{x_{d}} y_{d}^{1-\alpha} d y_{d}=\frac{1}{(1+\alpha)(2-\alpha)} x_{d}^{2-\alpha} .
$$

Furthermore we can show more easily that the other terms of $R_{2}$ are $o\left(x_{d}^{2}\right)$, In fact, by $|y|^{d+\alpha} \geq|\tilde{y}|^{d-1+\alpha_{\epsilon}}\left|y_{d}\right|^{1-\epsilon}$,

$$
\int_{|\tilde{y}|<1} d \tilde{y} \int_{0}^{x_{d}}\left(x_{d}^{2}+y_{d}^{2}\right)|\tilde{y}|^{2} \frac{d y_{d}}{|y|^{d+\alpha}} \leq \int_{|\tilde{y}|<1} \frac{|\tilde{y}|^{2}}{|\tilde{y}|^{d-1+\alpha_{\epsilon}}} d \tilde{y} \int_{0}^{x_{d}}\left(x_{d}^{2}+y_{d}^{2}\right) \frac{d y_{d}}{y_{d}^{1-\epsilon}}=C x_{d}^{2+\epsilon} .
$$

Therefore we have $R_{2}(x) \leq C x_{d}^{2-\alpha}$ for all $0<\alpha<2$.

In the second term of the right hand side of (4.2), we divide the integral area to $\{|y| \geq 1\},\{|y|<1\}$ and denote the corresponding terms by $S_{1}(x), S_{2}(x)$ respectively. For $S_{1}$, by

$$
\left|f(x+y)-f\left(\tilde{y}+\tilde{x}, y_{d}-x_{d}\right)\right| \leq 2 x_{d}\left\|\partial_{d} f\right\|_{\infty},
$$

we have

$$
S_{1}(x) \leq\left(2 x_{d}\left\|\partial_{d} f\right\|_{\infty} \cdot 3\|f\|_{\infty}+2 C x_{d}^{2}\right) \int_{|y| \geq 1} \frac{d y_{d}}{|y|^{d+\alpha}} \leq C x_{d} .
$$

For $S_{2}$, by (4.3) and by $|f(x)| \leq C x_{d}$, 


$$
\begin{aligned}
& \left|\left\{f(x+y)-f\left(\tilde{y}+\tilde{x}, y_{d}-x_{d}\right)\right\}\left\{f(x+y)+f\left(\tilde{y}+\tilde{x}, y_{d}-x_{d}\right)-2 f(x)\right\}+2 f(x)^{2}\right| \\
& \quad \leq 2\left\|\partial_{d} f\right\|_{\infty} x_{d} \cdot C\left(x_{d}+y_{d}\right)+2 C x_{d}^{2} \\
& \quad \leq C x_{d}\left(x_{d}+y_{d}\right) .
\end{aligned}
$$

Hence, noting that $\{|y|<1\} \subset\{|\tilde{y}|<1\} \times\left\{\left|y_{d}\right|<1\right\}$,

$$
\left|S_{2}(x)\right| \leq C x_{d} \int_{|\tilde{y}|<1} d \tilde{y} \int_{x_{d}}^{1}\left(x_{d}+y_{d}\right) \frac{d y_{d}}{|y|^{d+\alpha}} \leq 2 C x_{d} \int_{|\tilde{y}|<1} d \tilde{y} \int_{x_{d}}^{1} y_{d} \frac{d y_{d}}{|y|^{d+\alpha}} .
$$

By the same way as in $R_{2}$, we can show the desired estimate as follows. Let

$$
\int_{x_{d}}^{1} d y_{d} y_{d}\left(\int_{|\tilde{y}|<y_{d}}+\int_{y_{d} \leq|\tilde{y}|<1}\right) \frac{d \tilde{y}}{|y|^{d+\alpha}}=:\left(S_{2,1}(x)+S_{2,2}(x)\right) .
$$

Then $\quad\left|S_{2}(x)\right| \leq C x_{d}\left(S_{2,1}(x)+S_{2,2}(x)\right)$. Let $\quad 0<\epsilon<2-\alpha$. By $\quad|y|^{d+\alpha} \geq$ $|\tilde{y}|^{d-1-\epsilon}\left|y_{d}\right|^{1+\alpha_{\epsilon}}$,

$$
S_{2,1}(x) \leq \int_{x_{d}}^{1} \frac{d y_{d}}{y_{d}^{1+\alpha_{\epsilon}}} y_{d} \int_{|\tilde{y}|<y_{d}} \frac{d \tilde{y}}{|\tilde{y}|^{d-1+\epsilon}}=\int_{x_{d}}^{1} \frac{d y_{d}}{y_{d}^{\alpha+\epsilon}} \frac{y_{d}^{\epsilon}}{\epsilon}=\frac{1}{\epsilon} \int_{x_{d}}^{1} \frac{d y_{d}}{y_{d}^{\alpha}} .
$$

That is, if $0<\alpha<1$, then $S_{2,1}(x) \leq C$, if $\alpha=1$, then $S_{2,1}(x) \leq C \log \left(1 / x_{d}\right)$, if $0<\alpha<1$, then $S_{2,1}(x) \leq C x_{d}^{1-\alpha}$. Moreover for $S_{2,2}$, as in $R_{2,2}$, by $\int_{y_{d} \leq|\tilde{y}|<1} d \tilde{y} /|\tilde{y}|^{d+\alpha} \leq y_{d}^{-1-\alpha} /(1+\alpha)$,

$$
S_{2,2}(x) \leq \int_{x_{d}}^{1} d y_{d} y_{d} \int_{y_{d} \leq|\tilde{y}|<1} \frac{d \tilde{y}}{|\tilde{y}|^{d+\alpha}} \leq \int_{x_{d}}^{1} y_{d} \frac{1}{1+\alpha} y_{d}^{-1-\alpha} d y_{d}=\frac{1}{1+\alpha} \int_{x_{d}}^{1} y_{d}^{-\alpha} d y_{d} .
$$

Thus $S_{2,2}$ satisfies the same estimates as $S_{2,1}$. By $\left|S_{2}(x)\right| \leq C x_{d}\left(S_{2,1}(x)+S_{2,2}(x)\right)$, we have if $0<\alpha<1$, then $S_{2}(x) \leq C x_{d}$, if $\alpha=1$, then $S_{2}(x) \leq C x_{d} \log \left(1 / x_{d}\right)$ if $1<\alpha<2$, then $S_{2}(x) \leq C x_{d}^{2-\alpha}$. These imply our desired result.

By $P_{t}^{-} C_{c}^{\infty} \subset C_{p, 0}^{3}$, the following result for martingale problem is obtained by the same way as in $d=1$.

THEOREM 6. Let $\mu \in \mathscr{M}_{g_{p, 0}}$. The martingale problems for $\left(\mathscr{L}_{0}, \mathscr{D}_{0}, \mu\right)$, $\left(\mathscr{L}^{Y}, \mathscr{D}_{0}, \mu\right)$ associated with absorbing stable motion on $H$ are well-posed.

\section{Acknowledgment}

The author would like to be grateful to the referee for his suggestions and comments. 


\section{References}

[ 1 S. Hiraba, Infinite Markov particle systems with singular immigration; Martingale problems and limit theorems, Osaka J. Math. 33 (1996), 145-187.

[2] S. Hiraba, Independent infinite Markov particle systems with jumps, Theory Stoch. Process. 18 (34) (2012), 65-85.

\section{Department of Mathematics}

Faculty of Science and Technology

Tokyo University of Science

2641 Yamazaki, Noda City

Chiba 278-8510, Japan

E-mail: hiraba_seiji@ma.noda.tus.ac.jp 